\title{
BMP9 overexpressing adipose-derived mesenchymal stem cells promote cartilage repair in osteoarthritis-affected knee joint via the Notch1/Jagged1 signaling pathway
}

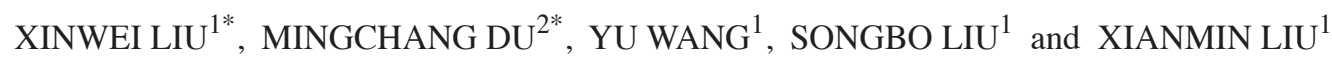 \\ ${ }^{1}$ Department of Orthopedics and Rescue Center of Severe Wound and Trauma of Chinese PLA, \\ General Hospital of Shenyang Military Area Command of Chinese PLA, Shenyang, Liaoning 110016; \\ ${ }^{2}$ Department of Orthopedics, Orthopedic Hospital of Shenyang, Shenyang, Liaoning 110000, P.R. China
}

Received November 26, 2017; Accepted August 20, 2018

DOI: $10.3892 /$ etm.2018.6754

\begin{abstract}
Osteoarthritis (OS) is a common disease in orthopedics. Although OS is known as an inflammation mediated by inflammatory cytokines; however, the mechanism is poorly understood. In the present study, the role of bone morphogenetic protein-9 (BMP9) was investigated in chondrogenic differentiation of adipose-derived mesenchymal stem cells (ADMSCs). ADMSCs were transfected with BMP9. BMP9 mRNA expression was detected by reverse transcription-quantitative polymerase chain reaction (RT-qPCR). Type II collagen and aggrecan expression was detected by western blotting and RT-qPCR. Mouse models of knee OS were established. Hematoxylin-eosin staining and toluidine blue staining were performed to observe changes in the OS-affected knee joint. After intra-articular injection of ADMSCs transfected with BMP9, intra-articular expression of type II collagen and aggrecan was detected by western blot analysis and RT-qPCR . After the Notch signaling pathway was inhibited in ADMSCs, ADMSCs were injected into the articular cavity. The expression of Notch signaling pathway-related proteins Notch1 and Jagged1 was detected by western blot analysis and RT-qPCR . BMP9 promoted chondrogenic differentiation of ADMSCs.
\end{abstract}

Correspondence to: Dr Xianmin Liu, Department of Orthopedics and Rescue Center of Severe Wound and Trauma of Chinese PLA, General Hospital of Shenyang Military Area Command of Chinese PLA, 83 Wenhua Road, Shenyang, Liaoning 110016, P.R. China E-mail: luming1956@sina.com

*Contributed equally

Abbreviations: BMP9, bone morphogenetic protein-9; OS, osteoarthritis; MSCs, mesenchymal stem cells; ADMSCs, adipose-derived mesenchymal stem cells; BMMSCs, bone marrow mesenchymal stem cells; TGF- $\beta$, transforming growth factor- $\beta$; GDF-2, growth differentiation factor 2

Key words: osteoarthritis, BMP9, adipose-derived mesenchymal stem cells, Notch1/Jagged1 signaling pathway
After injection of BMP9 overexpressing ADMSCs into the articular space, type II collagen and aggrecan expression was increased. When the Notch signaling pathway of ADMSCs was inhibited, the ability of BMP9 overexpressing ADMSCs to repair the cartilage in the OS-affected knee joint was attenuated. These results demonstrate that upregulating BMP9 protein expression may promote the chondrogenic differentiation of ADMSCs. Intra-articular injection of ADMSCs contributes to cartilage repair in OS-affected knee joints through the Notch1/Jagged1 signaling pathway.

\section{Introduction}

Osteoarthritis (OS) is a common disease in orthopedics with a high incidence in the middle-aged and elderly individuals (1). Pathologically, it is mainly characterized by loss of cartilage and degenerative changes (2). Cartilage tissue has a poor self-repair ability due to insufficient blood supply and lack of lymphatic vessel and nerve tissue distribution. The main symptoms of OS are pain and limited mobility, which affect a patient's normal life and place a heavy burden on families and society. Artificial joint replacement is a relatively effective treatment method of OS (3). To a certain degree, it eases patient's pain, but this surgery yields many short-term and long-term complications such as severe trauma, high costs, potential infection, loosening and sinking of joint implant. Cartilage transplantation, periosteal transplantation, and chondrocyte transplantation have not achieved satisfactory therapeutic effects, and there are many shortcomings such as limited donor source, immune rejection, and potential implant degradation. Therefore, their clinical application has remained limited. It is imperative to find a more simple and effective treatment.

Mesenchymal stem cells (MSCs), which are derived from the mesoderm, possess multi-directional differentiation potential (4). Under different induction conditions, MSCs can differentiate into osteoblasts (5), chondroblasts (6), and adipocytes (7). MSCs have been widely used in tissue engineering and regenerative medicine $(8,9)$. A previous study has demonstrated that intra-articular injection of MSCs can repair cartilage defects in OS patients (10). Adipose-derived MSCs (ADMSCs) 
have gradually become a new generation of cells for tissue engineering because they possess the advantages of abundant donor source and being easily accessible over bone marrow-derived MSCs (BMMSCs) as well as no concerns about the ethical issue present in use of embryonic stem cells or harvesting difficulty like that encountered in obtaining BMMSCs. MSCs differentiation into articular chondrocytes is the key to treatment of cartilage defects in OS. Chondrogenic differentiation of MSCs contributes to repair of cartilage defects.

Bone morphogenetic proteins (BMPs), belonging to transforming growth factor- $\beta$ (TGF- $\beta$ ) superfamily, is named because of their ability to induce the formation of bone and cartilage. More than 20 BMPs have been known, in which 15 BMPs are human BMPs (11). BMP2, BMP4 and BMP7 exhibit a strong ability to induce osteogenesis, and BMP2 and BMP7 have been widely used in the clinic (12-14). However, BMP2 and BMP7 induced non-fusion spine occasionally occur in the clinic $(15,16)$. BMP9, also known as growth differentiation factor 2 (GDF-2), is the protein with the strongest ability to induce chondrogenic differentiation among the BMPs family (17). BMP9 can be obtained from the liver of mice (18). In addition to inducing chondrogenic differentiation, BMP9 also has the ability to induce and maintain cholinergic differentiation of embryonic neurons (19), inhibit the production of hepatic glucose, promote the metabolism of fatty acids (20), stimulate hepcidin-1 expression, and thereby regulate iron homeostasis in vivo (21). BMP9 exhibits a variety of biological functions and has been widely concerned because of its induction of chondrogenic differentiation. However, the mechanism by which BMP9 induces chondrogenic differentiation remains poorly understood. Whether BMP9 can be used as a cytokine for bone regeneration remains to be a hot issue.

Many signaling pathways are involved in the process of chondrogenic differentiation of ADMSCs, such as the Notch signaling pathway, Wnt signaling pathway, and TGF- $\beta$ signaling pathway (22-24). The Notch signaling pathway is closely related to ADMSCs differentiation and organ formation and it is a key regulator of cell fate. There is evidence that during embryogenesis, the Notch signaling pathway is essential for the development of limb cartilage and bone (25). The Notch signaling pathway is also involved in the chondrogenict differentiation of MSCs in vitro (26). But the precise mechanism remains unclear. The majority of previous studies mainly investigated the change of the Notch signaling pathway during chondrogenic differentiation (27). There are no studies on actively regulating the Notch signaling pathway for chondrogenic differentiation of MSCs. At the same time, the mutual effects of BMP9 and the Notch signaling pathway in the chondrogenic differentiation of ADMSCs are unclear. In this study, we investigated the mutual effects of BMP9 and the Notch signaling pathway during the chondrogenic differentiation of ADMSCs through regulating BMP9 expression and the Notch signaling pathway. We also established mouse models of OS to investigate the role of BMP9 and the Notch signaling pathway in the repair of cartilage in OS affected knee joint using ADMSCs.

\section{Materials and methods}

Animals and cells. Female Balb/c mice, weighing 23-25 g, aged 8 weeks, were purchased from Beijing Vital River
Laboratory Animal Technology Co., Ltd. (Beijing, China). Mouse were raised a normal diet and water, raising conditions: ambient temperature of $20-26^{\circ} \mathrm{C}$, relative humidity of $40-70 \%$, alternating day and night time of 12/12 h. Mouse ADMSCs were purchased from Cyagen Biological Technology Co., Ltd. (Taicang, China; MUBMD-01001). Animal experiments were performed in Department of Laboratory Animals, General Hospital of Shenyang Military Region (Shenyang, China; license no. SYXK2015002). The experiments were approved by Animal Ethics Committee of General Hospital of Shenyang Military Region (no. 2015049).

Groups. Mice were randomly divided into five groups, with eight mice in each group: Sham, OS, OS + ADMSCs (MSCs), OS + ADMSCs + BMP9 (BMP9), and OS + LY411575 + ADMSCs + BMP9 (LY) groups. In the sham group, mice were not subjected to any procedure. In the OS group, OS was induced. In the MSCs group, OS was induced, and ADMSCs were injected into the articular cavity. In the BMP9 group, OS was induced, and BMP9 overexpressing ADMSCs were injected into the articular cavity. In the LY group, OS was induced, and LY411575 $(1.5 \mu \mathrm{mol} / \mathrm{l})(28,29)$ was injected into the articular cavity to inhibit the Notch signaling pathway, and BMP9 overexpressing ADMSCs were also injected.

In the cell experiment, ADMSCs were divided into four groups: Control group (only ADMSCs), induced group (induced chondrogenic differentiation of ADMSCs), BMP9 group (chondrogenic induced ADMSCs overexpress BMP9), and LY411575 (LY) group (the Notch signaling pathway was inhibited by LY411575 (ab142164; Abcam, Cambridge, UK) at a final concentration of $1 \mathrm{nM}$ (30), and chondrogenic induced ADMSCs overexpressed BMP9).

Chondrogenic induction of ADMSCs. ADMSCs in the logarithmic grow th phase were digested. Cell suspension at a density of $1 \times 10^{5} / 1$ was seeded into a 6 -well plate. Chondrogenic medium DMEM (C11885500BT; Gibco; Thermo Fisher Scientific, Inc., Waltham, MA, USA) supplemented with $10 \mathrm{ng} / \mathrm{ml}$ TGF- $\beta 1$, $50 \mathrm{nM}$ vitamin $\mathrm{C}, 6.25 \mathrm{mg} / \mathrm{l}$ insulin, and $10 \%$ fetal bovine serum (SH30068.03; Hyclone; GE Healthcare Life Sciences, Logan, UT, USA) was added and refreshed every 2 days. After 3 days of culture, cells were collected. Type II collagen and aggrecan expression in the chondrogenic induced ADMSCs was detected by PCR and western blot assay.

BMP9-transfected ADMSCs. ADMSCs in the logarithmic growth phase at a final concentration of $1 \times 10^{5} / 1$ were cultured with DMEM supplemented with pVSV-G-BMP9 (GenePharma Co., Ltd., Shanghai, China) and Polybrene (5 $\mu \mathrm{g} / \mathrm{ml})$. After $12 \mathrm{~h}$, culture medium was refreshed and cells were cultured for 2 more days. Cells were observed under fluorescence microscope. BMP9-transfected ADMSCs were identified by real-time fluorescence quantitative PCR method.

Establishment of mouse models of OS. Mouse models of OS were established by transection of the knee anterior cruciate ligament (31). Precisely, after anesthesia, an anesthesia ventilataor was used. The flow rate of isoflurane was adjusted. A medial patellar incision was made and the skin and articular capsule were cut open. The patella was laterally 
retracted. The knee was flexed as far as possible to expose the anterior cruciate ligament and the anterior horn of the medial meniscus. The bilateral anterior cruciate ligament was transected. The anterior drawer experiment was performed to confirm that the bilateral anterior cruciate ligament was completely cut off. Caution should be made to protect articular cartilage surface. The articular space was flushed with normal saline. Articular capsule and skin were sutured layer by layer.

Intra-articular injection of ADMSCs. ADMSCs were digested and prepared into cell suspension (at a cell density of $1 \times 10^{7} / \mathrm{ml}$ ) using chondrogenic medium DMEM (C11885500BT; Gibco; Thermo Fisher Scientific, Inc.). Four weeks after OS induction, ADMSCs were injected into the articular capsule once a week. Precisely, after anesthesia, a $2 \mathrm{~mm}$-long lateral incision was made on the lower limb. The knee was touched by stretching the skin. Cell suspension was injected into the articular cavity via the midpoint of the medial edge of the ligament. $10 \mu \mathrm{l}$ ADMSCs per articular cavity was injected. After needle withdrawal, the entry site was slightly pressurized to prevent the overflow of cell suspension. Skin incision was sutured.

$H \& E$ staining. Four weeks after intra-articular injection of ADMSCs, knee joint was disarticulated and fixed with formalin. The harvested tissue sample was de-calcified, dehydrated and embedded with paraffin. Sample tissues were de-waxed, rehydrated, stained with hematoxylin for $5 \mathrm{~min}$, washed with PBS, differentiated with hydrochloric acid ethanol for $3 \mathrm{sec}$, stained with eosin for $3 \mathrm{~min}$, dehydrated, cleared, mounted with neutral resin, and finally observed under the optical microscope (NE950; Leica Microsystems, Inc., Buffalo Grove, IL, USA).

Toluidine blue staining. Tissue sections $(5 \mu \mathrm{m})$ were de-waxed by xylene, rehydrated, and stained with $0.1 \%$ toluidine blue for 10 min at room temperature, washed with PBS three times, differentiated with glacial acetic acid, dehydrated in ethanol gradients, cleared with clear liquid, dried, and mounted with neutral gum.

Western blot analysis. Total protein was extracted from ADMSCs and knee tissue samples in different groups. Protein concentration was determined using a BCA protein assay kit (23227; Thermo Fisher Scientific, Inc.). Protein samples were subjected to SDS-PAGE and then transferred to a PVDF membrane. After addition of type II collagen (ab34712), aggrecan (ab3778), notch1 (ab52627), Jagged1 (ab7771), and GAPDH (ab181602; all Abcam) protein samples were incubated at $4^{\circ} \mathrm{C}$ overnight and washed with PBS. After addition of secondary antibody (goat anti-rabbit IgG/HRP antibody; 1:2,000; Bioss, Beijing, China), protein samples were incubated at $37^{\circ} \mathrm{C}$ for $2 \mathrm{~h}$. Protein bands were visualized using an ECL chemiluminescence detection kit (32109; Thermo Fisher Scientific, Inc.) and a gel imaging system (ChemiDoc MP; Bio-Rad Laboratories, Inc., Hercules, CA, USA). Absorbance analysis was performed using Image J software (Image J 1.8.0; National Institutes of Health, Bethesda, MD, USA).

Reverse transcription-quantitative polymerase chain reaction (RT-qPCR). Primers were designed according to the sequences of BMP9, type II collagen, aggrecan reported in Genbank, and were synthesized in Shanghai Biomedical Biotechnology Co., Ltd. Total RNA was isolated with TRIzol reagent (15596018), and reversely transcribed into cDNA (4387406; both Invitrogen; Thermo Fisher Scientific, Inc.). Real-time PCR kit (RR820A; Takara Biotechnology Co., Ltd., Dalian, China) was used for the detection. The relative gene expression data was analyzed with the $2^{-\Delta \Delta \mathrm{Cq}}$ method (32). The primers used for real-time PCR were listed as follows: BMP9 forward, GCTGCAGAACTG GGAACA and reverse, AACAAGCATCCCCTGGGG; Collagen II forward, TGCTGGCCCAACTGGCAA and reverse, ATTGTTGGTCTGCCTGGT; Aggrecan forward, CCA GTGAGGACCTGGTAGTG and reverse, CAGGCCTGCATG CACACCG; Notch1 forward, AAGAGGCTTGAGATGCTCC and reverse, TGCCTCAGCACACCGTGT; Jagged1 forward, TAACACCTTCAATCTCAAG and reverse, ATG ACACTATTCAACCTGA; GAPDH forward, GAATCGATC CATACTTATC and reverse, CCTTGAAGATATGGGCAC.

Statistical analysis. All data were statistically analyzed using SPSS v 19.0 software (IBM Corp., Armonk, NY, USA). A Kolmogorov-Smirnov (K-S) test was used to determine whether data were normally distributed. If data were normally distributed, the data were presented as mean \pm SEM. One-way ANOVA followed by a Student-Newman-Keuls test was used to test for differences among more than two groups. A level of $\mathrm{P}<0.05$ was considered to indicate a statistically significant difference.

\section{Results}

Chondrogenic induction of ADMSCs. To determine the differentiation of ADMSCs into chondrocytes, as confirmed by western blot assay (Fig. 1A) and RT-qPCR (Fig. 1B), after chondrogenic induction of ADMSCs, type II collagen and aggrecan expression levels were significantly increased in induced group ( $\mathrm{P}<0.05$ vs. control group), indicating that ADMSCs can be induced toward chondrogenic differentiation.

BMP9 promoted chondrogenic differentiation of ADMSCs. To determine the effect of BMP9, we performed BMP9 overexpression. At $72 \mathrm{~h}$ after pVSV-G-BMP9 lentivirus-transfected ADMSCs were directed toward chondrogenic differentiation, RT-qPCR was performed to detect intracellular BMP9 expression. After BMP9 transfection, intracellular BMP9 expression was significantly increased $(\mathrm{P}<0.05$; Fig. $2 \mathrm{~A}$ and $\mathrm{B})$, this result proved that our transfection was successful. We further detected the expression of type II collagen and aggrecan using western blot assay (Fig. 2C) and RT-qPCR (Fig. 2D). The results showed that type II collagen and aggrecan expression was significantly increased in BMP9-transfected ADMSCs than that in the chondrogenic induced ADMSCs $(\mathrm{P}<0.05)$. These results suggest that BMP9 promoted chondrogenic differentiation of ADMSCs possibly through the Notch signaling pathway.

BMP9 regulated the Notch1/Jaggedl signaling pathway to promote the chondrogenic differentiation of ADMSCs. In order to further explore the mechanism of BMP9, western blot assay was performed to detect the expression of Notch1/Jagged1 

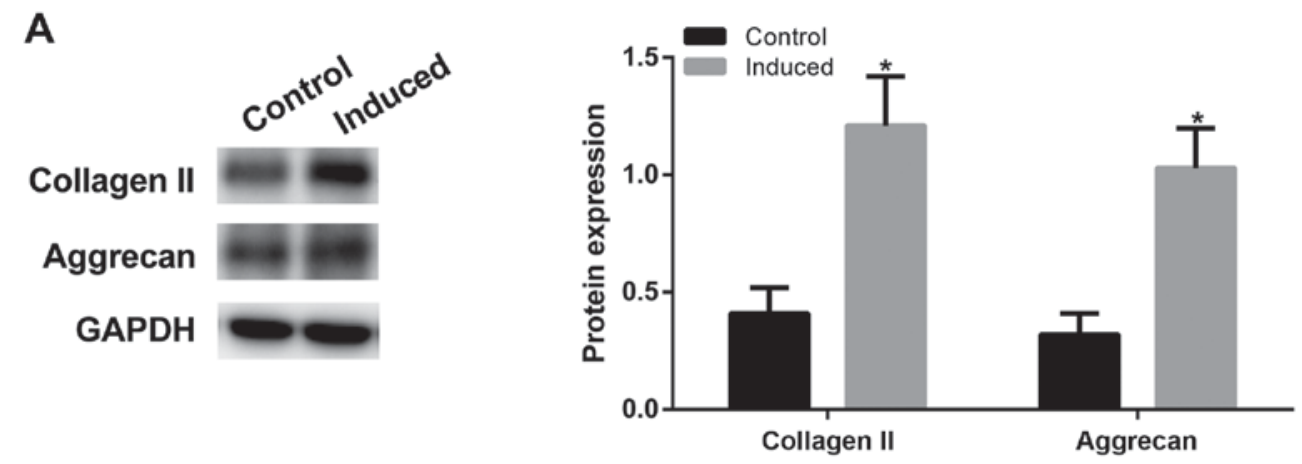

B

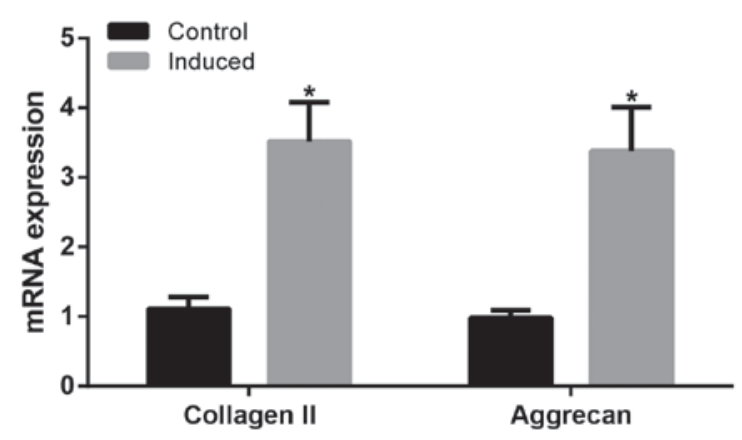

Figure 1. Chondrogenic induction of ADMSCs. ADMSCs induced cartilage cells, western blot assay and RT-qPCR detected the collagen II and Aggrecan expression. (A) Western blot assay. (B) RT-qPCR detection. Compared with control group, " $\mathrm{P}<0.05$. ADMSC, adipose-derived mesenchymal stem cells.

signaling pathway-related proteins Notch1 and Jagged1. After BMP9 was overexpressed, Notch1 and Jagged1 expression was significantly decreased than that in the chondrogenic induced ADMSCs ( $\mathrm{P}<0.05$; Fig. 3A). This was consistent with RT-qPCR findings (Fig. 3B). These results confirm that BMP9 regulated chondrogenic differentiation of ADMSCs through the Notch1/Jagged1 signaling pathway.

ADMSCs promoted cartilage repair in OS affected joints in mice. $\mathrm{H} \& \mathrm{E}$ staining results (Fig. 4) showed that in the OS group, articular chondrocytes were poorly arranged, and their number was smaller than that in sham group, and cartilage was thinner than that in the control group. Toluidine blue staining (Fig. 4B) showed that in the OS group, proteoglycan was unevenly distributed. After intra-articular injection of BMP9 overexpressing ADMSCs, the number of chondrocytes in the articular cavity was increased, and cartilage was thickened. In order to further explore the role of BMP9 in mice, western blot assay (Fig. 4C) was performed to detect intra-articular expression of type II collagen and aggrecan protein. The results showed that after BMP9 overexpression, type II collagen and aggrecan expression were significantly increased. When the Notch signaling pathway in the ADMSCs was inhibited, type II collagen and aggrecan expression were significantly decreased. This was consistent with RT-qPCR findings (Fig. 4D). These results demonstrate that BMP9 overexpressing ADMSCs can promote the healing of OS in mice.

BMP9 regulated the Notch/Jaggedl signaling pathway in ADMSCs to promote OS healing in mice. To further validate whether BMP9 overexpressing ADMSCs function effect via the Notch1/Jagged1 signaling pathway, western blot assay was performed to detect intra-articular expression of notch1 and Jagged1. In the BMP9 group, intra-articular expression of Notch1 and Jagged1 was significantly higher than that in the OS group $(\mathrm{P}<0.05)$ (Fig. 5A). When the Notch signaling pathway was inhibited, type II collagen and aggrecan expression was decreased (Fig. 4C and D) and Jagged1 expression was also significantly reduced ( $\mathrm{P}<0.05$ vs. BMP9 group). This was supported by RT-qPCR findings (Fig. 5B). These results confirmed that BMP9 overexpressing ADMSCs promote OS healing in mice through the Notch/Jagged1 signaling pathway.

\section{Discussion}

Stem cells are mainly obtained from skeletal muscle satellite cells, embryonic stem cells, and bone marrow MSCs (BMMSCs). It is difficult to obtain skeletal muscle satellite cells because of its lower level. Embryonic stem cells have immunological rejections and ethical issues. BMMSCs also have the shortcomings of difficult harvesting and that patients are not willing to accept.

Zuk et al (33) were the first to harvest ADMSCs with multi-directional differentiation potential from adipose tissue suspension during human liposuction and induced them to differentiate into adipocytes, chondroblasts, osteoblasts, and neural progenitors. ADMSCs are easily accepted because of easily accessible rich resource, being able to rapidly proliferate in any kind of serum, no immunological considerations during autografting, and no ethical issues. At present, there are no specific surface markers of ADMSCs. Gronthos and Zannettino (34) cultured cells of fat tissue aspirates, systematically studied cell surface markers, and found that 
A

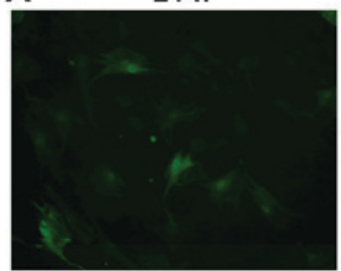

C
$48 \mathrm{~h}$
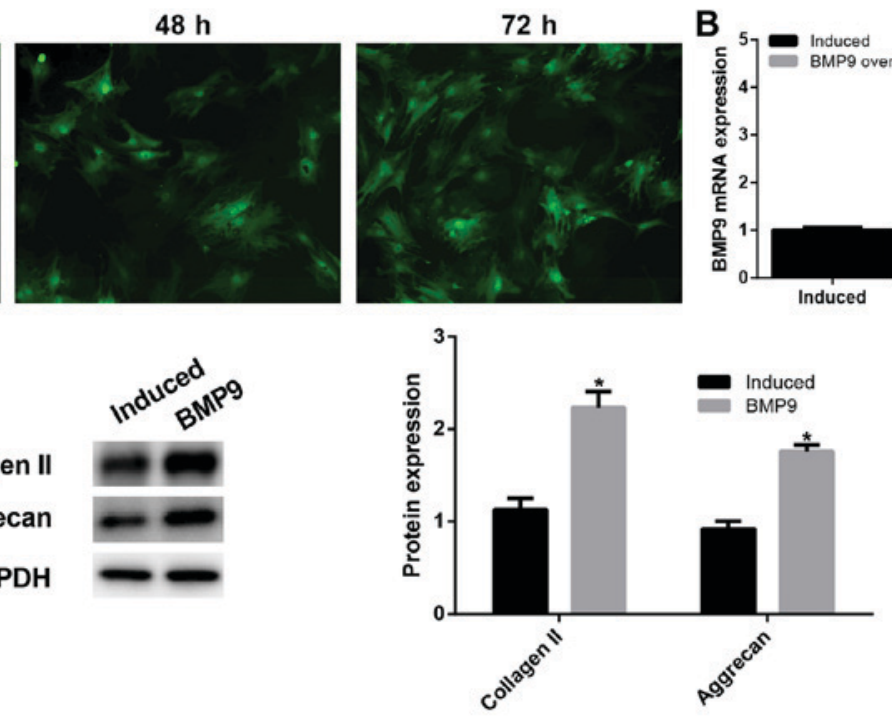
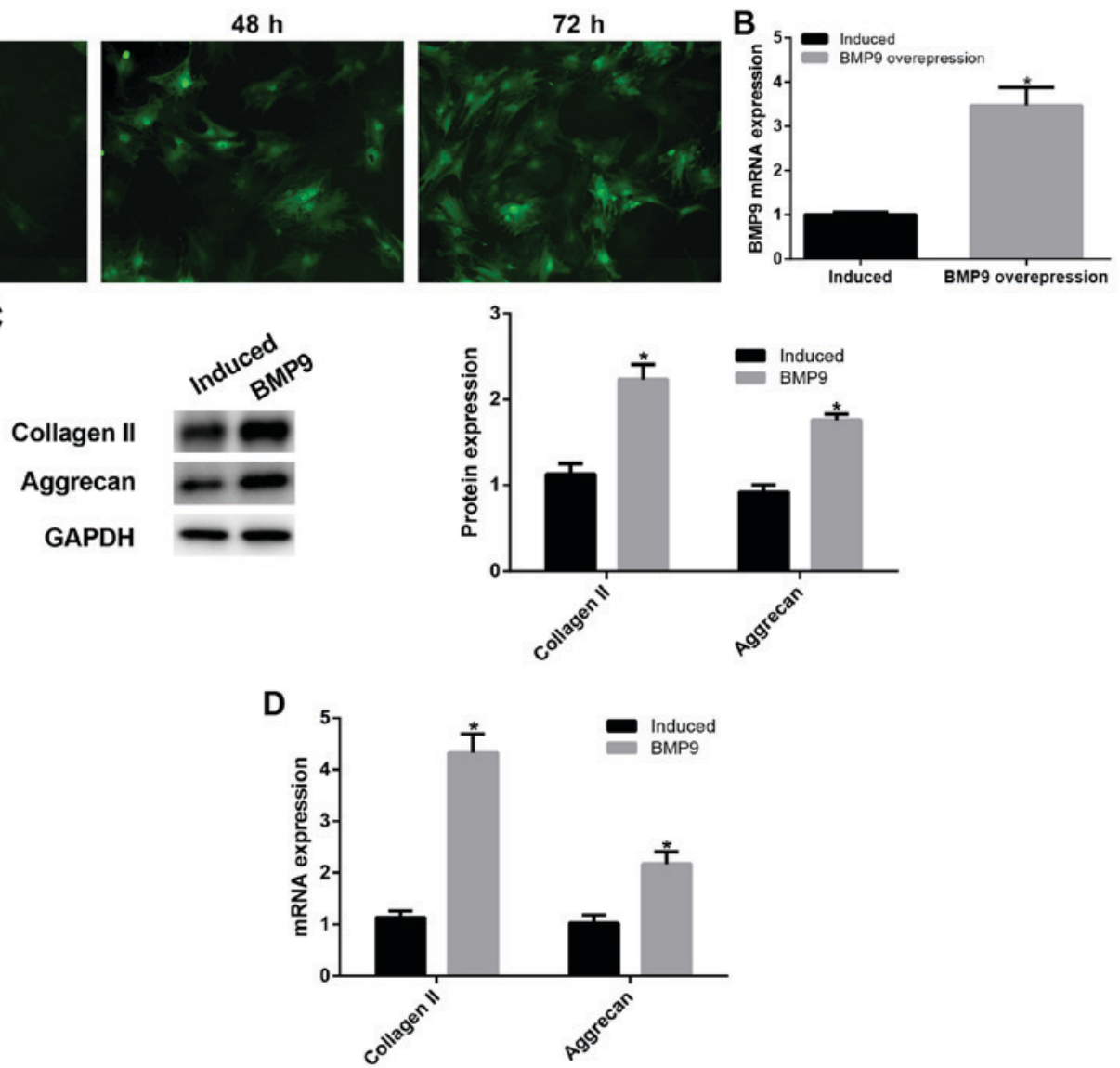

Figure 2.BMP9 promoted chondrogenic differentiation of ADMSCs. (A) Fluorescence signal intensity 24,48 and $72 \mathrm{~h}$ after pVSV-G-BMP9 lentivirus-transfected ADMSCs (magnification, x500), (B) Determination of the transfection of BMP9, RT-qPCR was performed to detect intracellular BMP9 expression. (C and D) Detection of expression of type II collagen and aggrecan after BMP9 transfected into ADMSCs by western blot assay and RT-qPCR, respectively. Compared with Induced group, ${ }^{\mathrm{P}}<0.05$. ADMSC, adipose-derived mesenchymal stem cells; BMP9, bone morphogenetic protein-9.

A

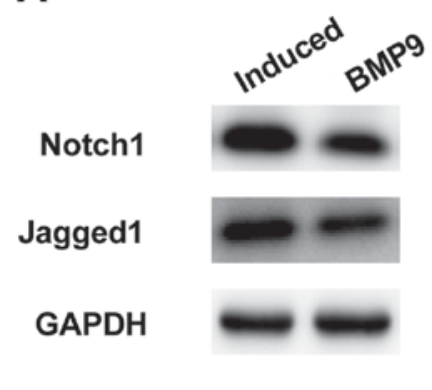

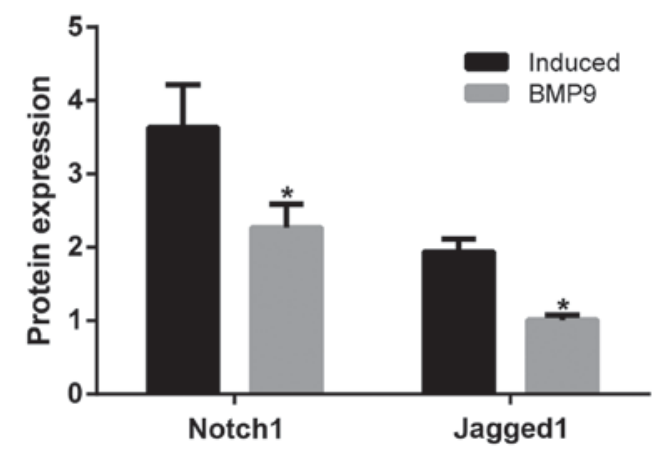

B

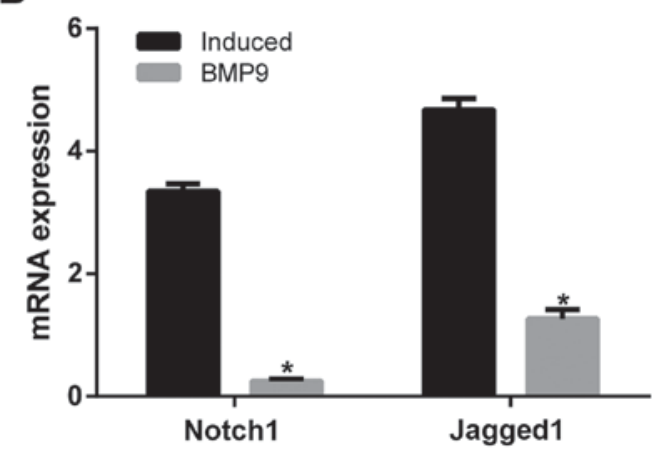

Figure 3. BMP9 regulated the Notch/Jagged1 signaling pathway promotes the chondrogenic differentiation of ADMSCs. (A) Western blot assay for detecting the Notch/Jagged1 signaling pathway related protein expression, and determining the relationship between BMP9 and Notch pathway. (B) RT-qPCR detection. Compared with Induced group, ${ }^{*} \mathrm{P}<0.05$. BMP9, bone morphogenetic protein-9. 
A
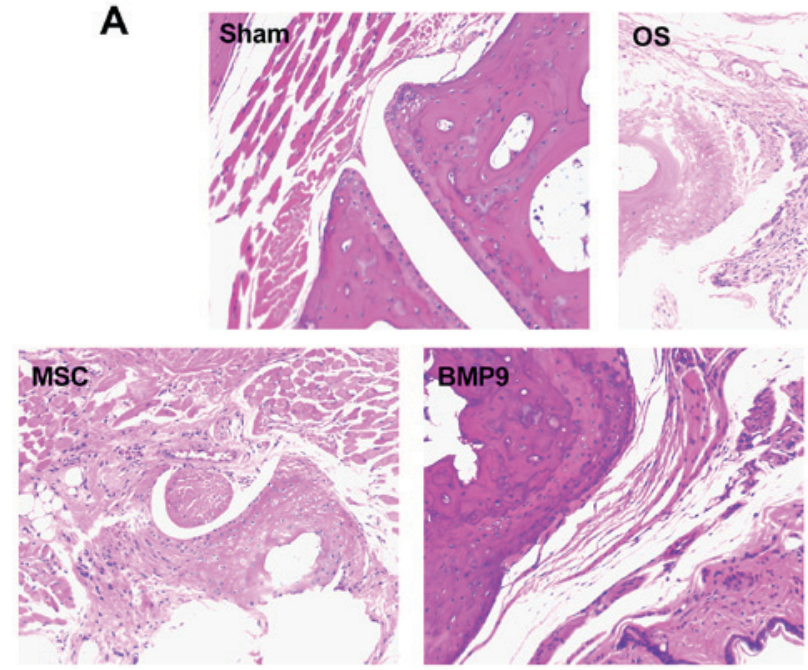
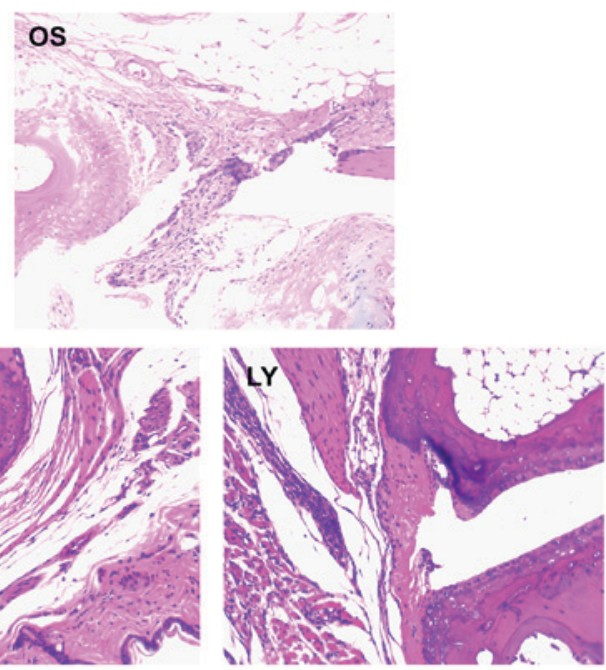

B
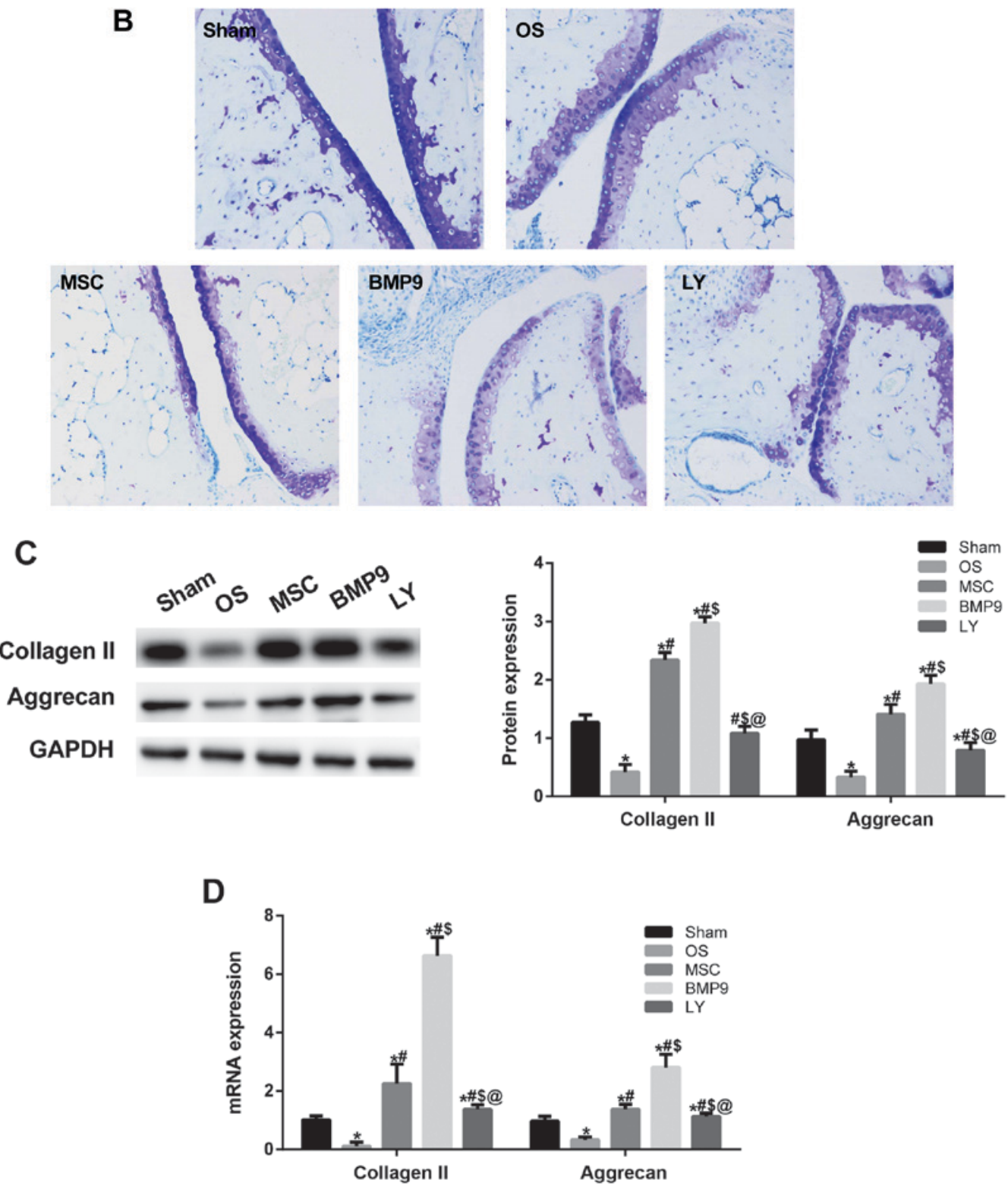

Figure 4. ADMSCs promoted cartilage repair in OS affected joints in mice. After OS model was established, ADMSCs were injected into joint cavity, and the changes of joint were observed by H\&E staining and toluidine blue staining. Western blot assay and RT-qPCR were used to detect the collagen II and Aggrecan expression. (A) H\&E staining (magnification, x400). (B) Toluidine blue staining (magnification, x400). (C) Western blot assay. (D) RT-qPCR detection. Compared with sham group, ${ }^{\mathrm{P}} \mathrm{P}<0.05$; Compared with OS group, ${ }^{\text {}} \mathrm{P}<0.05$; Compared with MSC group, ${ }^{\$} \mathrm{P}<0.05$; Compared with BMP9 group, ${ }^{\circledR} \mathrm{P}<0.05$. BMP9, bone morphogenetic protein-9; OS, osteoarthritis; MSC, mesenchymal stem cells; ADMSC, adipose-derived MSC. 
A
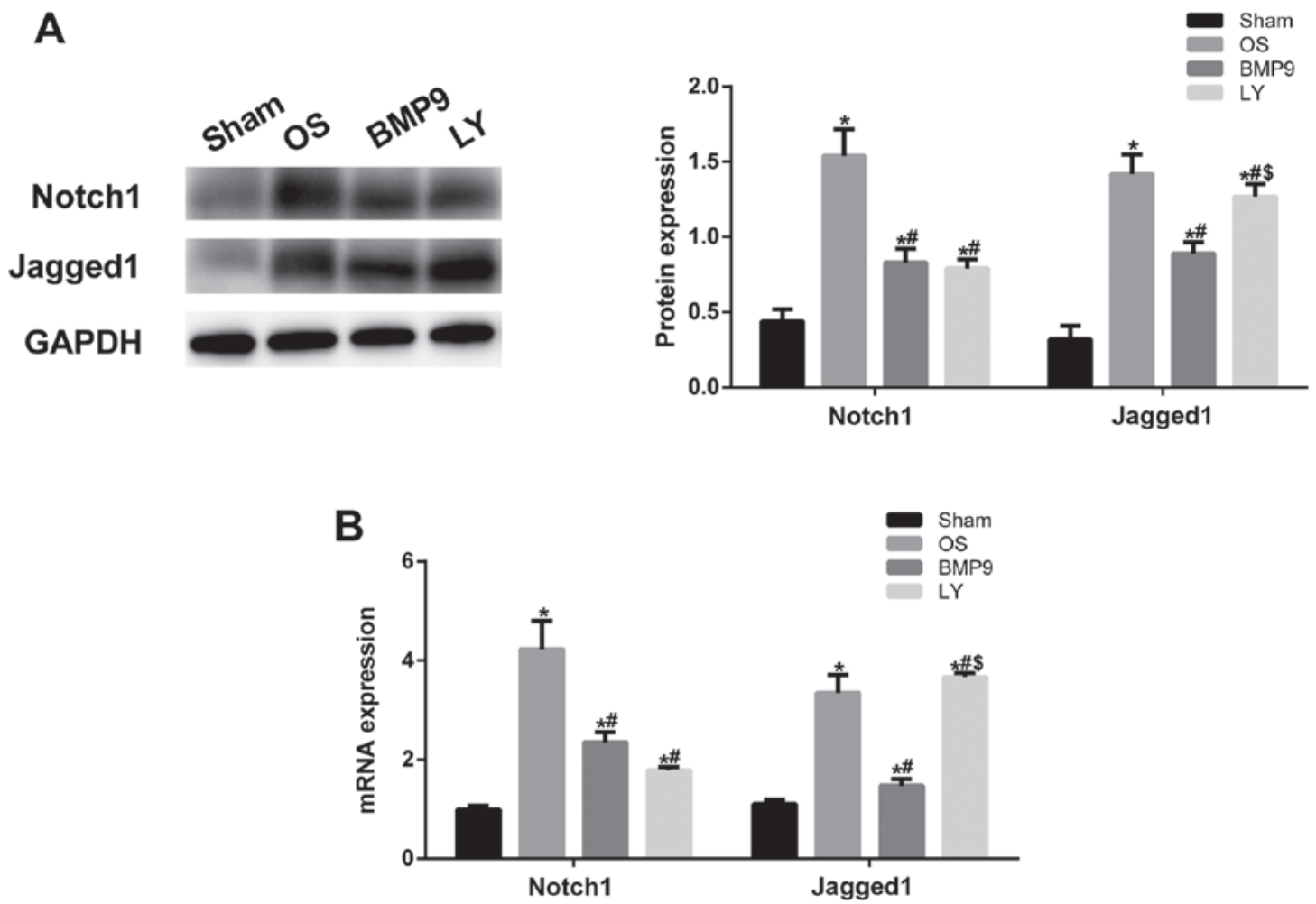

Figure 5. BMP9 overexpressing ADMSCs promote OS healing in mice by regulating the Notch/Jagged1 signaling pathway. After OS model was established, ADMSCs were injected into joint cavity, western blot assay and RT-qPCR were utilized to detect Notch signaling pathway related protein expression. (A) Western blot assay. (B) RT-qPCR detection. Compared with sham group, ${ }^{*} \mathrm{P}<0.05$; Compared with OS group, ${ }^{\text {"P }}<0.05$; compared with BMP9 group, ${ }^{\$} \mathrm{P}<0.05$. BMP9, bone morphogenetic protein-9; OS, osteoarthritis; ADMSC, adipose-derived mesenchymal stem cells.

ADMSCs were spindle-shaped, had abundant cytoplasm and nucleoli, and grew in a parallel or spiral-like manner. These cells have surface markers, including CD59, CD105, CD106, CD146 and CD165, which are similar to BMMSCs (35). But STRO-1 antigen was not detected on ADMSCs.

Festy et al (36) found that ADMSCs are similar to fully differentiated adipocyte surface markers, and the surface markers are not different between subcutaneous ADMSCs and omental ADMSCs. In the experiments, ADMSCs were isolated. Cell surface markers CD13, CD44 and CD59 on ADMSCs were detected. Flow cytometry showed that CD13, CD44, and CD59 were positive, indicating that the harvested ADMSCs are highly purified. ADMSCs, as a kind of multi-potential stem cells, share the features with stem cells, that is to say, ADMSCs theoretically have the ability to infinitely proliferate.

BMP9, as a less studied BMP, has a very strong potential for chondrogenesis. There is evidence that BMP9 together with the Notch signaling pathway plays an important role in embryonic development, cell proliferation and differentiation, and the occurrence of diseases (37). In the early study, BMP9 and the Notch signaling were found to have synergistic effects in the early stage of osteogenesis (38). However, the precise mechanism remains poorly understood. This is the problem that needs to be studied in this paper.

In this study, we overexpressed BMP9 in ADMSCs, observed change in cell masses under the inverted microscope, and detected cartilage type II collagen and aggrecan expression. Results showed that upregulating BMP9 expression can further induce chondrogenic differentiation of ADMSCs. To further clarify the mutual effects of the Notch signaling pathway and
BMP9 in the chondrogenic differentiation of ADMSCs, we used LY411575 to inhibit the Notch signaling pathway. Inhibiting the Notch signaling pathway using LY411575 can inhibit the chondrogenic differentiation of ADMSCs, confirming that the Notch signaling pathway can inhibit the chondrogenic differentiation of ADMSCs.

After intra-articular injection of ADMSCs, a larger degree of cartilage repair was found in the MSCs group than in the control group. After intra-articular injection of BMP9 overexpressing ADMSCs, type II collagen and aggrecan protein expression in the cartilage of OS affected knee joint was further detected by immunohistochemical staining to further confirm the mechanism underlying BMP9-overexpressing ADMSCs. BMP9 overexpressing ADMSCs were injected into the articular cavity to inhibit the Notch signaling pathway. Results showed that ADMSCs promoted OS healing in mice through the Notch1/Jagged1 signaling pathway.

Taken together, ADMSCs express multiple stem cell surface markers and can be induced to differentiate into chondrocytes, confirming that ADMSCs exhibit multi-directional differentiation potential. Upregulating BMP9 protein can promote the chondrogenic differentiation of ADMSCs. Inhibition of the Notch signaling pathway can inhibit the chondrogenic differentiation of ADMSCs. Intra-articular injection of ADMSCs contributes to cartilage repair OS affected knee joint in mice, and the repair is achieved via the Notch1/Jagged1 signaling pathway.

\section{Acknowledgements}

Not applicable. 


\section{Funding}

The present study was supported by a grant from the Liaoning Province Science and Technology Issues in China (grant no. 2013225089).

\section{Availability of data and materials}

The datasets used and/or analyzed during the current study are available from the corresponding author on reasonable request.

\section{Authors' contributions}

XinL and XiaL conceived and designed the study, acquired data, interpreted the results and drafted the manuscript. XiaL also contributed to acquisition of funding support. MD, YW and SL performed the experiments. XinL and XiaL analyzed the data. All authors read and approved the final manuscript.

\section{Ethics approval and consent to participate}

The experiments were approved by Animal Ethics Committee of General Hospital of Shenyang Military Region, China (no. 2015049).

\section{Patient consent for publication}

Not applicable.

\section{Competing interests}

The authors declare that they have no competing interests.

\section{Reference}

1. Kobayashi T, Takagishi K, Shitara H, Ichinose T, Shimoyama D, Yamamoto A, Osawa T and Tajika T: Prevalence of and risk factors for shoulder osteoarthritis in Japanese middle-aged and elderly populations. J Shoulder Elbow Surg 23: 613-619, 2014.

2. Nuki G: Osteoarthritis: A problem of joint failure. Z Rheumatol 58: 142-147, 1999.

3. Ding S and Zheng K: Artificial total hip arthroplasty with collum femoris preserving for treating hip joint. Zhongguo Xiu Fu Chong Jian Wai Ke Za Zhi 24: 1-4, 2010 (In Chinese).

4. Komaki M, Iwasaki K and Morita I: Bone and stem cells. Mesenchymal stem cells and bone regeneration. Clin Calcium 24: 565-573, 2014.

5. Endo I and Mastumoto T: Bone and stem cells. Regulatory mechanism of mesenchymal stem cell differentiation to osteoblasts. Clin Calcium 24: 555-564, 2014.

6. Nishimura R, Nakamura E, Kida J, Yagi H and Hata K: Bone and stem cells. Regulation of chondrocyte differentiation from mesenchymal stem cells. Clin Calcium 24: 509-516, 2014.

7. Hamam D, Ali D, Vishnubalaji R, Hamam R, Al-Nbaheen M, Chen L, Kassem M, Aldahmash A and Alajez NM: microRNA-320/RUNX2 axis regulates adipocytic differentiation of human mesenchymal (skeletal) stem cells. Cell Death Dis 5: e1499, 2014

8. Sanz AR, Carrión FS and Chaparro AP: Mesenchymal stem cells from the oral cavity and their potential value in tissue engineering. Periodontol 2000 67: 251-267, 2015.

9. Park JS, Suryaprakash S, Lao YH and Leong KW: Engineering mesenchymal stem cells for regenerative medicine and drug delivery. Methods 84: 3-16, 2015.

10. Wang Y, Yuan M, Guo QY, Lu SB and Peng J: Mesenchymal stem cells for treating articular cartilage defects and osteoarthritis. Cell Transplant 24: 1661-1678, 2015.
11. Wozney JM: Overview of bone morphogenetic proteins. Spine (Phila Pa 1976) 27 (16 Suppl 1): S2-S8, 2002.

12. Zhang X, Guo J, Wu G and Zhou Y: Effects of heterodimeric bone morphogenetic protein-2/7 on osteogenesis of human adipose-derived stem cells. Cell Prolif 48: 650-660, 2015.

13. Duan Z, Zheng Q, Guo X, Yuan Q and Chen S: Experimental research on ectopic osteogenesis of BMP2-derived peptide P24 combined with PLGA copolymers. J Huazhong Univ Sci Technolog Med Sci 27: 179-182, 2007.

14. Choi YJ, Lee JY, Park JH, Park JB, Suh JS, Choi YS, Lee SJ, Chung CP and Park YJ: The identification of a heparin binding domain peptide from bone morphogenetic protein-4 and its role on osteogenesis. Biomaterials 31: 7226-7238, 2010.

15. Werle S, AbuNahleh K and Boehm H: Bone morphogenetic protein 7 and autologous bone graft in revision surgery for non-union after lumbar interbody fusion. Arch Orthop Trauma Surg 136: 1041-1049, 2016.

16. Mladenov KV, Kunkel P and Stuecker R: The use of recombinant human BMP-2 as a salvage procedure in the pediatric spine: A report on 3 cases. Eur Spine J 19 (Suppl 2): S135-S139, 2010.

17. Tang N, Song WX, Luo J, Luo X, Chen J, Sharff KA, Bi Y, He BC, Huang JY, Zhu GH, et al: BMP-9-induced osteogenic differentiation of mesenchymal progenitors requires functional canonical Wnt/beta-catenin signalling. J Cell Mol Med 13: 2448-2464, 2009.

18. Breitkopf-Heinlein K, Meyer C, König C, Gaitantzi H, Addante A, Thomas M, Wiercinska E, Cai C, Li Q, Wan F, et al: BMP-9 interferes with liver regeneration and promotes liver fibrosis. Gut 66: 939-954, 2017

19. López-Coviella I, Berse B, Krauss R, Thies RS and Blusztajn JK: Induction and maintenance of the neuronal cholinergic phenotype in the central nervous system by BMP-9. Science 289: 313-316, 2000

20. Luo Y, Li L, Xu X, Wu T, Yang M, Zhang C, Mou H, Zhou T, Jia Y, Cai C, et al: Decreased circulating BMP-9 levels in patients with Type 2 diabetes is a signature of insulin resistance. Clin Sci (Lond) 131: 239-246, 2017.

21. Truksa J, Peng H, Lee P and Beutler E: Different regulatory elements are required for response of hepcidin to interleukin-6 and bone morphogenetic proteins 4 and 9. Br J Haematol 139: 138-147, 2007.

22. Fortini C, Cesselli D, Beltrami AP, Bergamin N, Caragnano A, Moretti L, Cecaro F, Aquila G, Rizzo P, Riberti C, et al: Alteration of Notch signaling and functionality of adipose tissue derived mesenchymal stem cells in heart failure. Int J Cardiol 174: 119-126, 2014.

23. Fang J, Wei Y, Lv C, Peng S, Zhao S and Hua J: CD61 promotes the differentiation of canine ADMSCs into PGC-like cells through modulation of TGF- $\beta$ signaling. Sci Rep 7: 43851, 2017.

24. Cui X, He Z, Liang Z, Chen Z, Wang $\mathrm{H}$ and Zhang J: Exosomes from adipose-derived mesenchymal stem cells protect the myocardium against ischemia/reperfusion injury through Wnt $/ \beta$-catenin signaling pathway. J Cardiovasc Pharmacol 70: 225-231, 2017.

25. Hosaka Y, Saito T, Sugita S, Hikata T, Kobayashi H, Fukai A, Taniguchi Y, Hirata M, Akiyama H, Chung UI and Kawaguchi H: Notch signaling in chondrocytes modulates endochondral ossification and osteoarthritis development. Proc Natl Acad Sci USA 110: 1875-1880, 2013.

26. Chen AX, Hoffman MD, Chen CS, Shubin AD, Reynolds DS and Benoit DS: Disruption of cell-cell contact-mediated Notch signaling via hydrogel encapsulation reduces mesenchymal stem cell chondrogenic potential: Winner of the society for biomaterials student award in the undergraduate category, charlotte, NC, April 15 to 18,2015. J Biomed Mater Res A 103: 1291-1302, 2015.

27. Matthews BG, Grcevic D, Wang L, Hagiwara Y, Roguljic H, Joshi P, Shin DG, Adams DJ and Kalajzic I: Analysis of aSMA-labeled progenitor cell commitment identifies Notch signaling as an important pathway in fracture healing. J Bone Miner Res 29: 1283-1294, 2014.

28. Kramer J, Schwanbeck R, Pagel H, Cakiroglu F, Rohwedel J and Just U: Inhibition of Notch signaling ameliorates acute kidney failure and downregulates platelet-derived growth factor receptor $\beta$ in the mouse model. Cells Tissues Organs 201: 109-117, 2016.

29. Ortiz-Martínez F, Gutiérrez-Aviñó FJ, Sanmartín E, Pomares-Navarro E, Villalba-Riquelme C, García-Martínez A, Lerma E and Peiró G: Association of Notch pathway down-regulation with triple negative/basal-like breast carcinomas and high tumor-infiltrating FOXP3+ tregs. Exp Mol Pathol 100: 460-468, 2016. 
30. Chen J, Chang H, Peng X, Gu Y, Yi L, Zhang Q, Zhu J and Mi M: 3,6-dihydroxyflavone suppresses the epithelial-mesenchymal transition in breast cancer cells by inhibiting the Notch signaling pathway. Sci Rep 6: 28858, 2016.

31. Lorenz $\mathrm{J}$ and Grässel S: Experimental osteoarthritis models in mice. Methods Mol Biol 1194: 401-419, 2014.

32. Livak KJ and Schmittgen TD: Analysis of relative gene expression data using real-time quantitative PCR and the 2(-Delta Delta C(T)) method. Methods 25: 402-408, 2001.

33. Zuk PA, Zhu M, Mizuno H, Huang J, Futrell JW, Katz AJ, Benhaim P, Lorenz HP and Hedrick MH: Multilineage cells from human adipose tissue: Implications for cell-based therapies. Tissue Eng 7: 211-228, 2001.

34. Gronthos S and Zannettino AC: Methods for the purification and characterization of human adipose-derived stem cells. Methods Mol Biol 702: 109-120, 2011.

35. Gronthos S, Franklin DM, Leddy HA, Robey PG, Storms RW and Gimble JM: Surface protein characterization of human adipose tissue-derived stromal cells. J Cell Physiol 189: 54-63 2001 .
36. Festy F, Hoareau L, Bes-Houtmann S, Péquin AM, Gonthier MP, Munstun A, Hoarau JJ, Césari M and Roche R: Surface protein expression between human adipose tissue-derived stromal cells and mature adipocytes. Histochem Cell Biol 124: 113-121, 2005.

37. Liu P, Man Y, Wang Y and Bao Y: Mechanism of BMP9 promotes growth of osteosarcoma mediated by the Notch signaling pathway. Oncol Lett 11: 1367-1370, 2016.

38. Liao J, Wei Q, Zou Y, Fan J, Song D, Cui J, Zhang W, Zhu Y, $\mathrm{Ma} \mathrm{C}, \mathrm{Hu} \mathrm{X}$, et al: Notch signaling augments BMP9-induced bone formation by promoting the osteogenesis-angiogenesis coupling process in mesenchymal stem cells (MSCs). Cell Physiol Biochem 41: 1905-1923, 2017.

(i) $($ This work is licensed under a Creative Commons Attribution-NonCommercial-NoDerivatives 4.0 International (CC BY-NC-ND 4.0) License. 